Egyptian

Orthodontic Journal

\title{
THE EFFECT OF $\beta$-THALASSEMIA ON MATURATION OF CERVICAL VERTEBRAE AND CRANIOFACIAL MORPHOLOGY
}

\author{
EMAN M. EL-SHOURBAGY*
}

ABSTRACT:

This study was performed to determine the effect of $\beta$-thalassemia on the cervical vertebrae maturation and craniofacial morphology of the affectd children. Twenty $\beta$-thalassemic patients $(10 \hat{0}, 10$ O ) ranging in age between 7 to 11 years and matching control sample were chosen for this study. The data were compiled from lateral cephalograms. The cervical vertebrae maturation stages were assessed by the Hassel and Farman modification of Lamparski's criteria. This method was able to detect delayed skeletal maturation in thalassemic patients when compared to the matching control. Cervical vertebrae measurements and craniofacial linear and angular parameters were recorded and statistically analyzed to detect any significant difference between the studied groups. The results revealed that, there was significant decrease in cervical vertebrae measurements of thalassemic patients with strong positive correlation among these measurements. At the same time, certain linear and angular craniofacial parameters of thalassemic patients displayed significant difference in comparison with the matching control. It is important to consider these variations during diagnosis and treatment plan.

\section{INTRODUCTION}

Thalassemia refers to a groups of hereditary hemolytic anemias that involve defects in synthesis of either alpha or beta chains of hemoglobin ${ }^{1,2}$.

*Associate Professor, Orthodontic Department, Faculty of Dentistry, Tanta University 
Egyptian

Orthodontic Journal

The term major, intermediate and minor used in conjunction with $\beta$ thalassemia and usually refers to the degree of severity of the signs and symptoms ${ }^{3,4}$. $\beta$ - thalassemias are more frequently found in Mediterranean and black population ${ }^{5}$.

Thalassemia major is the homozygous form of $\beta$-thalassemia and is the most common type ${ }^{6}$. The onset of thalassemia major occurs during the first or second year of life and if not treated, the patient may not survive beyond the age of 5 years. Survival depends on repeated blood transfusion and iron chelation ${ }^{7}$.

By the second year of life, the child begins to develop a mongoloid appearance with prominent frontal and parietal bosses, marked overgrowth of the maxilla, prominence of the malar bone and typical bony changes. These changes, include marked generalized osteoporosis, thinning of the cortices and marked dilatation of the medullary cavities of the long bones as well as failure of pneumatization of the maxillary sinuses ${ }^{8,9}$.

Borgna - Pignatti et al ${ }^{10}$ found that skeletal maturation was delayed in $83 \%$ of males and $75 \%$ of females of thalassemic patients.

Kattamis ${ }^{11}$ reported that many thalassemic patients suffered from growth retardation and failure of puberty, while some patients showed normal growth and development.

The results of khaldi et $\mathrm{al}^{12}$ on 39 patients with $\beta$-thalassemia aged 14 mon. to 14 years identified 6 patients with growth failure while the rest had normal growth rate.

Ponte et $\mathrm{al}^{13}$ studied 32 homozygous thalassemic patients aged between 2 to 16 years. Their study showed that the growth is near to average until preadolescent period and decreased progressively with pubertal development.

Skeletal maturity can be assessed by means of a series of biologic indicators: increase in body height ${ }^{14}$, skeletal maturation of the hand and wrist ${ }^{15}$, dental development and eruption ${ }^{16}$, sexual maturation characteristics ${ }^{17}$ and cervical vertebral maturation ${ }^{18-20}$.

With respect to the cervical vertebral method, 6 stages corresponding to different maturational phases in cervical vertebrae can be identified during the pubertal period ${ }^{18}$. The 6 stages in cervical vertebrae maturation include observations before the peak, i.e. during the accelerative growth phase (stages 1 to 3 ) and observations after the peak i.e. during the decelerative phase of growth (stages 4 to 6) pubertal growth peak occur on average between vertebral stages 3 and 4 . 
Egyptian

Orthodontic Journal

The effectiveness of the cervical vertebrae as maturational indicator has been corroborated by Hassel and Farman ${ }^{21}$ and Garcia- Fernandez et $\mathrm{al}^{22}$ who found a high correlation between cervical vertebral maturation and skeletal maturation of the hand and wrist.

On reviewing the literature, little was found to be dealing with the cervical vertebrae as skeletal maturation indicator.

Therefore, it seems of interest to evaluate the craniofacial morphology and cervical vertebral maturation of thalassemic patients on the basis of a single cephalometric observation and without additional exposures.

\section{Materials and methods}

The sample used in this study was made up of $20 \beta$-thalassemic patients (10 males and 10 females). They were selected from the Hematology unit at Tanta university hospital and the following criteria were fulfilled:

- Aged from 7 to 11 years

- No previous orthodontic treatment

- Free from any other disease that could affect growth.

A matching control sample of Egyptian children with acceptable facial profile and occlusion were obtained from a primary school.

For each subject enrolled in this study, standardized lateral cephalogram was taken, traced on acetate paper with $0.5 \mathrm{~mm}$ diameter lead pencil.

For evaluation of measurement error, all measurements were double checked and recorded by the investigator on different occasions. There was no significant difference between any of the variables using t-test at significant level of 0.05 .

Cervical vertebral development of the sample was evaluated by the Hassel and Farman ${ }^{21}$ modification of Lamparski's criteria which assesses maturational changes on the second, third and fourth cervical vertebrae .

Three parts of the cervical vertebrae were traced. These entities were the dens or odontoid process (CV2), the body of the third cervical vertebra (CV3) and the body of the fourth cervical vertebra (CV4). The cervical vertebrae tracings were checked on each lateral cephalometric radiograph to identify the presence or absence of curvature in the inferior borders of the CV2, CV3, CV4. General shapes of bodies of CV3, CV4, were evaluated, and assigned the stage which they most closely resembled. Stages of maturation of CV3 and CV4 bodies related to age group in this study were:1- Initiation 2- Acceleration 3-Transition (Fig.1). 
Some linear measurements were used for location of CV2, CV3, and CV4. Vertical perpendicular line was dropped from the Frankfort horizontal plane directly from porion, to this vertical line, measurements from the deepest point of the lower borders of CV2, CV3, and CV4, were made on a parallel to the Frankfort horizontal plane according to the method described by Bench ${ }^{22}$ (Fig.2).

The following cephalometric landmarks were identified for use in this study:

N (Nasion), S (Sella), A (Subspinale), AP Max (the anterior landmark for determining the length of the maxilla), B (Supramentale), Gn (Gnathion), Go (Gonion), Me (Mention) ,P (Anatomical porion), Ar (Articulare), Co (Condylion), Or (Orbitale), ANS (Anterior nasal spine) and PNS (Posterior nasal spine).

\section{Angular measurements used in this study (Fig.2):}

SNA

SNB

ANB

NSAr Saddle angle

SAr Go Articular angle

Ar Go Me Gonial angle

Sum Sum of saddle, articular and gonial angles

NS Gn Y axis

Pal Mp Basal plane angle

In addition to the cervical vertebrae measurements ( $\mathrm{P}-\mathrm{CV} 2, \mathrm{P}-\mathrm{CV} 3$, P- CV4 ), the following linear measurements were used (Fig.2) :

$\mathrm{N}$ ANS Upper anterior facial height

ANS Me Lower anterior facial height

$\begin{array}{lc}\text { N Me } & \text { Total Face height } \\ \text { S Go } & \text { Posterior facial height } \\ \text { S Gn } & \text { Y- axis } \\ \text { Co Gn } & \text { Total mandibular length } \\ \text { Co Go } & \text { Remus height } \\ \text { Go Gn } & \text { Extent of mandibular base } \\ \text { AP Max-PNS Extent of maxillary base . }\end{array}$




\section{Egyptian}

Orthodontic Journal
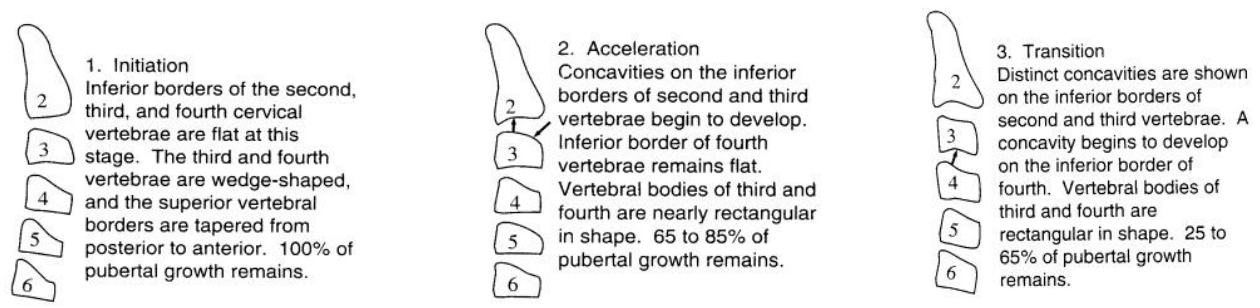

Fig. (1): Maturational stages of cervical vertebrae

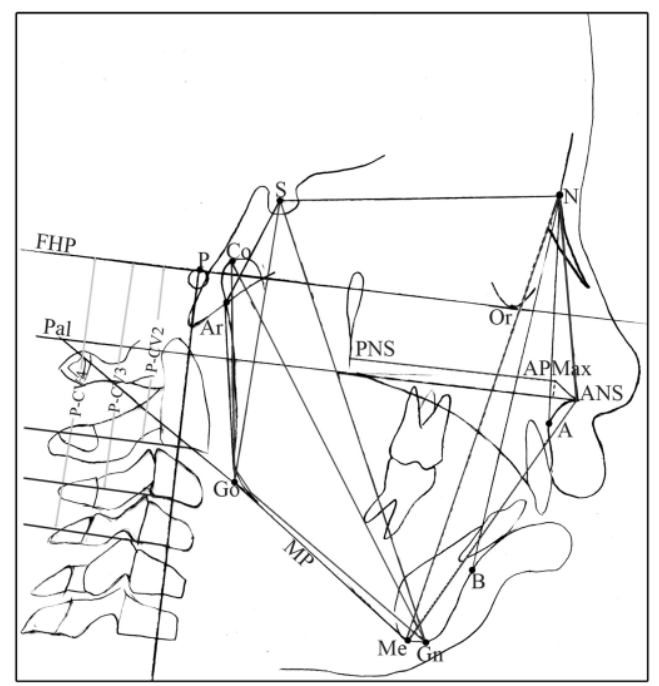

Fig. (2): Cephalometric landmarks and parameters used in this study

\section{Statistical analysis:}

The collected data were organized, tabulated and statistically analyzed using SPSS software statistical computer package version 12. For quantitative data, the range, mean and standard deviation were calculated. The difference between two means was statistically analyzed using the students $t$ test. Pearson's correlation coefficient (r) was calculated to test the association between two variables. For qualitative data, the number and percent distribution were 


\section{Egyptian}

Orthodontic Journal

calculated. Significance was adopted at $\mathrm{p}<0.05$ for interpretation of results of tests of significance ${ }^{24}$.

\section{RESULTS}

The results of the present study are shown in Tables 1-7 and Fig. 3-8.

The distribution of cervical vertebrae skeletal maturation stages of control subjects and thalassemic patients and their percentages according to Hassel and Farman $^{21}$ was summarized in Table 1 and Fig.3. Stage 1 was observed in 10 thalassemic patients $(5 \hat{O}, 5$ ㅇ $)$ and in one male control. Two thalassemic patients $(1 \hat{\jmath}, 1$ 우) and 2 male control subjects were observed between stages 1-2. Stage 2 was observed in 7 thalassemic patients $(4 \hat{\gamma}, 3$ + $)$ and in 11 control subjects $(6 \hat{\jmath}, 5+$.

Stage 3 of cervical vertebrae maturation was observed in one male patient and in 6 control subjects $(1 \hat{O}, 5 \bigcirc)$

As indicated by the results of this study, stage 1 through 2 occurred in the majority of thalassemic patients, while stage 2 through 3 occurred in the majority of control subjects.

Table (1): The prevalence of cervical vertebrae maturation stages of the studied groups and their percentage

\begin{tabular}{|l|l|l|l|l|l|l|l|l|}
\hline \multirow{2}{*}{ Staging } & \multicolumn{4}{|c|}{ Thalassemia } & \multicolumn{5}{c|}{ Control } \\
\cline { 2 - 10 } & \multicolumn{2}{|c|}{ Males } & \multicolumn{2}{|c|}{ Females } & \multicolumn{2}{c|}{ Males } & \multicolumn{2}{c|}{ Females } \\
\cline { 2 - 10 } & $\mathrm{n}$ & $\%$ & $\mathrm{n}$ & $\%$ & $\mathrm{n}$ & $\%$ & $\mathrm{n}$ & $\%$ \\
\hline $\mathbf{1}$ & 5 & 50.0 & 5 & 50.0 & 1 & 10.0 & 0 & 0.0 \\
\hline $\mathbf{1 - 2}$ & 1 & 10.0 & 1 & 10.0 & 2 & 20.0 & 0 & 0.0 \\
\hline $\mathbf{2}$ & 4 & 40.0 & 3 & 30.0 & 6 & 60.0 & 5 & 50.0 \\
\hline $\mathbf{2 - 3}$ & 0 & 0.0 & 0 & 0.0 & 0 & 0.0 & 0 & 0.0 \\
\hline $\mathbf{3}$ & 0 & 0.0 & 1 & 10.0 & 1 & 10.0 & 5 & 50.0 \\
\hline
\end{tabular}

Volume 27 - June 2005 
As demonstrated in Table 2, there were no meaningful sex difference between thalassemic patients for all computed variables except P-CV2 (Fig. 4), SGo, SGn and SNB.

Among controls (Table 3) males had significant greater mean in P-CV3 (Fig.5), ANS Me, NMe, SGn, AP Max PNS, Ar Go Me, sum of posterior angles, and Pal Mp as compared with the female subjects.

Comparisons between male thalassemic patients and control subjects demonstrated significant difference of all cervical vertebrae measurements (Fig. 6) and ANS Me, SGo, SGn, Co Go, Go Gn, SNA, ANB, NS Ar and SAr Go ( Table 4).

Where as in Table 5, a significant difference was detected between female thalassemic patientsand control as regards to P-CV3 (Fig.7), ANS Me, $\mathrm{N} \mathrm{Me}$, SGn, AP Max PNS, SNB, ANB, Ar Go Me, sum of the posterior angles and Pal $\mathrm{Mp}$. The rest of the variables showed no significant difference.

Table 6 presents the data for both sexes combined to increase the observed variance and the overlooking significant difference between thalassemic patients and the matching control. All variables demonstrated statistically significant differences except SNB, NS Gn. Fig.8 depicted the data concerning the cervical vertebrae.

In an attempt to find a correlation between the cervical vertebrae measurements and all variables used, it was noticed that all cervical vertebrae measurements positively correlated strongly with one other $(\mathrm{P}=0.001)$ Table 7.

A significant positive correlation was detected at $1 \%$ level of significance $(\mathrm{P}<0.01)$ between the following variables.

P-CV2: Go Gn, NS Ar

P-CV3: Go Gn, NS Ar

P-CV4: Go Gn, NS Ar

A significant positive correlation was found at $5 \%$ level of significance $(\mathrm{P}<0.05)$ between P-CV4 and Co Gn.

A significant negative correlation was found between the following variables at $1 \%$ level of significance:

P-CV2: ANB

P-CV3: ANB, Ar Go Me.

A significant negative correlation at 5\% level of significance was detected between the following:

P-CV2: SGn, SAr Go.

P-CV3: ANS Me, S Gn, SAr Go, NS Gn, Pal Mp.

P-CV4: ANB

Volume 27 - June 2005 
Egyptian

Orthodontic Journal

Table (2): Comparison of cervical vertebrae and craniofacial linear and angular parameters among patients with thalassemia.

\begin{tabular}{|c|c|c|c|c|c|c|c|c|}
\hline \multirow{2}{*}{ Variables } & \multicolumn{3}{|c|}{ Males } & \multicolumn{3}{|c|}{ Females } & \multirow{2}{*}{ t } \\
\cline { 2 - 8 } & Range & Mean & S.D. & Range & Mean & S.D. & & \\
\hline P-CV2 & $43-51$ & 46.20 & 2.94 & $46-54$ & 49.60 & 3.13 & 2.503 & $0.002^{* *}$ \\
\hline P-CV3 & $54-67$ & 59.60 & 4.55 & 57.67 & 61.30 & 3.30 & 0.956 & 0.352 \\
\hline P-CV4 & $66-81$ & 71.40 & 5.38 & $69-80$ & 75.00 & 3.74 & 1.737 & 0.099 \\
\hline \hline N ANS & $44-56$ & 50.0 & 4.37 & $45-58$ & 49.70 & 4.27 & 0.155 & 0.878 \\
\hline ANS Me & $63-79$ & 68.00 & 5.77 & 59.78 & 64.70 & 7.15 & 1.136 & 0.271 \\
\hline N Me & $105-126$ & 111.4 & 6.74 & $106-126$ & 113.0 & 7.47 & 0.503 & 0.621 \\
\hline S Go & $63-78$ & 71.80 & 5.09 & $62-71$ & 65.20 & 3.22 & 3.461 & $0.003 * *$ \\
\hline S Gn & $110-123$ & 119.6 & 4.99 & $105-123$ & 112.1 & 6.67 & 2.845 & $0.011 *$ \\
\hline Co Gn & $97-117$ & 107.4 & 6.75 & $95-116$ & 104.8 & 6.86 & 0.854 & 0.404 \\
\hline Co Go & $47-56$ & 53.10 & 3.35 & $47-56$ & 51.80 & 3.58 & 0.838 & 0.413 \\
\hline Go Gn & $60-69$ & 65.70 & 3.02 & $60-72$ & 65.90 & 3.38 & 0.139 & 0.891 \\
\hline AP Max- & $47-53$ & 49.90 & 2.18 & $42-53$ & 48.30 & 3.43 & 1.244 & 0.230 \\
\hline \hline PNS & $79-87$ & 83.70 & 2.79 & $76-86$ & 81.20 & 3.71 & 1.704 & 0.106 \\
\hline SNA & $74-81$ & 77.70 & 2.36 & $70-78$ & 74.90 & 2.85 & 2.395 & $0.028 *$ \\
\hline SNB & $3-10$ & 6.00 & 2.67 & $3-11$ & 6.20 & 2.49 & 0.173 & 0.864 \\
\hline ANB & $117-126$ & 121.4 & 3.20 & $120-134$ & 124.4 & 4.67 & 1.675 & 0.111 \\
\hline NS Ar & $139-151$ & 144.7 & 4.81 & $136-152$ & 145.2 & 6.00 & 0.206 & 0.839 \\
\hline S Ar Go & $127-133$ & 130.1 & 2.47 & $124-138$ & 131.4 & 5.46 & 0.686 & 0.502 \\
\hline Ar Go Me & $389-406$ & 396.4 & 5.83 & $386-409$ & 401.2 & 7.83 & 1.555 & 0.137 \\
\hline Sum & $65-75$ & 68.90 & 3.48 & $67-75$ & 71.20 & 3.01 & 1.581 & 0.131 \\
\hline NS Gn & $24-33$ & 28.00 & 3.02 & $22-42$ & 30.20 & 7.74 & 0.837 & 0.419 \\
\hline Pal Mp & & & & & & \\
\hline
\end{tabular}

* Sig at $0.05 \%$

** Sig at $0.01 \%$

Volume 27 - June 2005 
Egyptian

Orthodontic Journal

Table (3): Comparison of cervical vertebrae and craniofacial linear and angular parameters of control group in relation to sex.

\begin{tabular}{|c|c|c|c|c|c|c|c|c|}
\hline \multirow{2}{*}{ Variables } & \multicolumn{3}{|c|}{ Males } & \multicolumn{3}{|c|}{ Females } & \multirow{2}{*}{$\mathbf{t}$} & \multirow{2}{*}{$\mathbf{p}$} \\
\hline & Range & Mean & S.D. & Range & Mean & S.D. & & \\
\hline P-CV2 & $50-57$ & 53.80 & 2.10 & $48-57$ & 52.40 & 3.10 & 1.183 & 0.253 \\
\hline P-CV3 & $64-70$ & 67.00 & 1.76 & $62-67$ & 64.90 & 1.85 & 2.596 & $0.018^{*}$ \\
\hline P-CV4 & 73-83 & 78.00 & 2.87 & 75-78 & 77.00 & 1.05 & 1.035 & 0.314 \\
\hline N ANS & 46-49 & 47.40 & 1.17 & $45-.50$ & 47.20 & 1.69 & 0.308 & 0.762 \\
\hline ANS Me & $60-65$ & 62.50 & 1.43 & 56-61 & 58.00 & 1.76 & 6.260 & $0.001 * *$ \\
\hline N Me & 106-112 & 109.2 & 1.93 & 99-109 & 104.4 & 3.75 & 3.600 & $0.002 * *$ \\
\hline S Go & $64-66$ & 65.00 & 0.94 & $58-69$ & 65.00 & 4.00 & 0.000 & 1.000 \\
\hline S Gn & $107-114$ & 110.9 & 2.02 & $102-112$ & 106.6 & 4.12 & 2.965 & $0.011^{*}$ \\
\hline Co Gn & 98-108 & 102.9 & 4.09 & 99-105 & 101.9 & 2.08 & 0.689 & 0.503 \\
\hline Co Go & $44-51$ & 48.10 & 2.28 & $48-53$ & 49.50 & 1.72 & 1.550 & 0.138 \\
\hline Go Gn & $65-72$ & 68.30 & 2.26 & $65-72$ & 68.60 & 2.76 & 0.266 & 0.793 \\
\hline $\begin{array}{c}\text { AP Max- } \\
\text { PNS } \\
\end{array}$ & $45-52$ & 47.70 & 2.91 & $44-47$ & 45.50 & 1.08 & 2.243 & $0.046 *$ \\
\hline SNA & $79-82$ & 80.50 & 1.35 & $78-83$ & 80.60 & 1.78 & 0.142 & 0.889 \\
\hline SNB & $75-79$ & 77.40 & 1.35 & $75-80$ & 77.80 & 1.75 & 0.572 & 0.574 \\
\hline ANB & $2-4$ & 3.10 & 0.57 & $2-4$ & 2.80 & 0.79 & 0.976 & 0.342 \\
\hline NS Ar & $120-129$ & 126.4 & 2.72 & $124-130$ & 126.70 & 2.21 & 0.271 & 0.790 \\
\hline S Ar Go & 136-145 & 140.8 & 2.82 & 129-151 & 140.20 & 6.71 & 0.261 & 0.799 \\
\hline Ar Go Me & $120-131$ & 128.0 & 3.06 & $116-129$ & 123.90 & 4.77 & 2.288 & $0.034 *$ \\
\hline Sum & $388-402$ & 396.1 & 4.41 & $373-395$ & 390.60 & 6.43 & 2.230 & $0.039^{*}$ \\
\hline NS Gn & $66-70$ & 68.40 & 1.26 & $67-70$ & 68.40 & 1.17 & 0.000 & 1.000 \\
\hline Pal Mp & $25-30$ & 27.90 & 1.66 & $22-26$ & 23.90 & 1.45 & 5.734 & $0.001 * *$ \\
\hline
\end{tabular}

* Sig at $0.05 \%$

** Sig at $0.01 \%$

Volume 27 - June 2005 
Egyptian

Orthodontic Journal

Table(4) : Comparison of cervical vertebrae and craniofacial linear and angular parameters of male thalassemia and control.

\begin{tabular}{|c|c|c|c|c|c|c|c|c|}
\hline \multirow{2}{*}{ Variables } & \multicolumn{3}{|c|}{ Thalassemia patients } & \multicolumn{3}{|c|}{ Control } & \multirow{2}{*}{$\mathbf{t}$} & \multirow{2}{*}{$\mathbf{p}$} \\
\hline & Range & Mean & S.D. & Range & Mean & S.D. & & \\
\hline $\mathrm{P}-\mathrm{CV} 2$ & $43-51$ & 46.20 & 2.94 & $50-57$ & 53.80 & 2.10 & 6.660 & $0.001 * *$ \\
\hline $\mathrm{P}-\mathrm{CV} 3$ & $54-67$ & 59.60 & 4.55 & $64-70$ & 67.00 & 1.76 & 4.794 & $0.001 * *$ \\
\hline $\mathrm{P}-\mathrm{CV} 4$ & $66-81$ & 71.40 & 5.38 & $73-83$ & 78.00 & 2.87 & 3.424 & $0.003 * *$ \\
\hline "N ANS & 444-56 & ב50.0 & 4.37 & "46-49 & 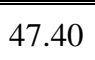 & 1.17 & 101.816 & 0.099 \\
\hline ANS Me & $63-79$ & 68.00 & 5.77 & $60-65$ & 62.50 & 1.43 & 2.924 & $0.015^{*}$ \\
\hline $\mathrm{N} \mathrm{Me}$ & $105-126$ & 111.4 & 6.74 & $106-112$ & 109.2 & 1.93 & 0.993 & 0.343 \\
\hline S Go & $63-78$ & 71.80 & 5.09 & $64-66$ & 65.00 & 0.94 & 4.150 & $0.002 * *$ \\
\hline S Gn & $110-123$ & 119.6 & 4.99 & $107-114$ & 110.9 & 2.02 & 5.106 & $0.001 * *$ \\
\hline Co Gn & $97-117$ & 107.4 & 6.75 & $98-108$ & 102.9 & 4.09 & 1.802 & 0.088 \\
\hline Co Go & $47-56$ & 53.10 & 3.35 & $44-51$ & 48.10 & 2.28 & 3.902 & $0.001 * *$ \\
\hline Go Gn & $60-69$ & 65.70 & 3.02 & $65-72$ & 68.30 & 2.26 & 2.178 & $0.043^{*}$ \\
\hline $\begin{array}{l}\text { AP Max- } \\
\text { PNS }\end{array}$ & $47-53$ & 49.90 & 2.18 & $45-52$ & 47.70 & 2.91 & 1.913 & 0.072 \\
\hline SNA & $79-87$ & 83.70 & 2.79 & $79-82$ & 80.50 & 1.35 & 3.262 & $0.006 * *$ \\
\hline SNB & $74-81$ & 77.70 & 2.36 & $75-79$ & 77.40 & 1.35 & 0.349 & 0.732 \\
\hline ANB & $3-10$ & 6.00 & 2.67 & $2-4$ & 3.10 & 0.57 & 3.364 & $0.007 * *$ \\
\hline NS Ar & $117-126$ & 121.4 & 3.20 & $120-129$ & 126.4 & 2.72 & 3.764 & $0.001 * *$ \\
\hline S Ar Go & $139-151$ & 144.7 & 4.81 & $136-145$ & 140.8 & 2.82 & 2.212 & $0.043 *$ \\
\hline $\mathrm{Ar}$ Go $\mathrm{Me}$ & $127-133$ & 130.1 & 2.47 & $120-131$ & 128.0 & 3.06 & 1.690 & 0.108 \\
\hline Sum & $389-406$ & 396.4 & 5.83 & $388-402$ & 396.1 & 4.41 & 0.130 & 0.898 \\
\hline NS Gn & $65-75$ & 68.90 & 3.48 & $66-70$ & 68.40 & 1.26 & 0.427 & 0.677 \\
\hline Pal Mp & $24-33$ & 28.00 & 3.02 & $25-30$ & 27.90 & 1.66 & 0.092 & 0.928 \\
\hline
\end{tabular}

$\begin{array}{llll}* & \text { Sig } & \text { at } & 0.05 \% \\ * * & \text { Sig } & \text { at } & 0.01 \%\end{array}$

Volume 27 - June 2005 
Egyptian

Orthodontic Journal

Table (5): Comparison of cervical vertebrae and craniofacial linear and angular parameters of female thalassemia and control.

\begin{tabular}{|c|c|c|c|c|c|c|c|c|}
\hline \multirow{2}{*}{ Variables } & \multicolumn{3}{|c|}{ Thalassemia patients } & \multicolumn{3}{|c|}{ Control } & \multirow{2}{*}{$\mathbf{t}$} & \multirow{2}{*}{$\mathbf{p}$} \\
\hline & Range & Mean & S.D. & Range & Mean & S.D. & & \\
\hline P-CV2 & $46-54$ & 49.60 & 3.13 & $48-57$ & 52.40 & 3.10 & 2.009 & 0.060 \\
\hline P-CV3 & 57.67 & 61.30 & 3.30 & $62-67$ & 64.90 & 1.85 & 3.007 & $0.009 * *$ \\
\hline P-CV4 & $69-80$ & 75.00 & 3.74 & $75-78$ & 77.00 & 1.05 & 1.627 & 0.134 \\
\hline N ANS & $45-58$ & 49.70 & 4.27 & $45-.50$ & 47.20 & 1.69 & 1.722 & 0.111 \\
\hline ANS Me & 59.78 & 64.70 & 7.15 & $56-61$ & 58.00 & 1.76 & 2.877 & $0.016^{*}$ \\
\hline N Me & $106-126$ & 113.0 & 7.47 & 99-109 & 104.4 & 3.75 & 3.255 & $0.006 * *$ \\
\hline S Go & $62-71$ & 65.20 & 3.22 & $58-69$ & 65.00 & 4.00 & 0.123 & 0.903 \\
\hline S Gn & $105-123$ & 112.1 & 6.67 & $102-112$ & 106.6 & 4.12 & 2.218 & $0.040^{*}$ \\
\hline Co Gn & $95-116$ & 104.8 & 6.86 & 99-105 & 101.9 & 2.08 & 1.279 & 0.228 \\
\hline Co Go & $47-56$ & 51.80 & 3.58 & $48-53$ & 49.50 & 1.72 & 1.830 & 0.090 \\
\hline Go Gn & $60-72$ & 65.90 & 3.38 & $65-72$ & 68.60 & 2.76 & 1.957 & 0.066 \\
\hline $\begin{array}{l}\text { AP Max - } \\
\text { PNS }\end{array}$ & $42-53$ & 48.30 & 3.43 & $44-47$ & 45.50 & 1.08 & 2.460 & $0.032 *$ \\
\hline SNA & $76-86$ & 81.20 & 3.71 & $78-83$ & 80.60 & 1.78 & 0.462 & 0.650 \\
\hline SNB & $70-78$ & 74.90 & 2.85 & $75-80$ & 77.80 & 1.75 & 2.744 & $0.013 *$ \\
\hline ANB & $3-11$ & 6.20 & 2.49 & $2-4$ & 2.80 & 0.79 & 4.123 & $0.001 * *$ \\
\hline NS Ar & $120-134$ & 124.4 & 4.67 & $124-130$ & 126.7 & 2.21 & 1.407 & 0.176 \\
\hline S Ar Go & $136-152$ & 145.2 & 6.00 & $129-151$ & 140.2 & 6.71 & 1.757 & 0.096 \\
\hline Ar Go Me & $124-138$ & 131.4 & 5.46 & $116-129$ & 123.9 & 4.77 & 3.271 & $0.004 * *$ \\
\hline Sum & $386-409$ & 401.2 & 7.83 & $373-395$ & 390.6 & 6.43 & 3.308 & $0.004 * *$ \\
\hline NS Gn & $67-75$ & 71.20 & 3.01 & $67-70$ & 68.40 & 1.17 & 2.740 & $0.018^{*}$ \\
\hline Pal Mp & $22-42$ & 30.20 & 7.74 & $22-26$ & 23.90 & 1.45 & 2.529 & $0.031 *$ \\
\hline
\end{tabular}

* Sig at $0.05 \%$

** Sig at $0.01 \%$

Volume 27 - June 2005 


\section{Egyptian}

Orthodontic Journal

Table (6): Comparison of cervical vertebrae and craniofacial linear and angular parameters between thalassemic and control groups (both sexes combined).

\begin{tabular}{|c|c|c|c|c|c|c|c|c|}
\hline \multirow{2}{*}{ Variables } & \multicolumn{3}{|c|}{ Thalassemia patients } & \multicolumn{3}{|c|}{ Control } & \multirow{2}{*}{$\mathbf{t}$} & \multirow{2}{*}{$\mathbf{p}$} \\
\hline & Range & Mean & S.D. & Range & Mean & S.D. & & \\
\hline P-CV2 & $43-54$ & 47.90 & 3.43 & $48-57$ & 53.10 & 2.67 & 5.345 & $0.001 * *$ \\
\hline P-CV3 & $54-67$ & 60.45 & 3.97 & $62-70$ & 65.95 & 2.06 & 5.501 & $0.001 * *$ \\
\hline P-CV4 & $66-81$ & 73.20 & 4.87 & $73-83$ & 77.50 & 2.16 & 3.606 & $0.001 * *$ \\
\hline N ANS & $44-58$ & 49.85 & 4.21 & $45-50$ & 47.30 & 1.42 & 2.568 & $0.017 *$ \\
\hline ANS Me & $59-79$ & 66.35 & 6.55 & $56-65$ & 60.25 & 2.79 & 3.833 & $0.001 * *$ \\
\hline N Me & $105-126$ & 112.2 & 6.97 & $99-112$ & 106.8 & 3.81 & 3.041 & $0.005 * *$ \\
\hline S Go & $62-78$ & 68.50 & 5.36 & $58-69$ & 65.00 & 2.83 & 2.584 & $0.015^{*}$ \\
\hline S Gn & $105-123$ & 115.9 & 6.91 & $102-114$ & 108.8 & 3.85 & 4.015 & $0.001 * *$ \\
\hline Co Gn & $95-117$ & 106.1 & 6.76 & 98-108 & 102.4 & 3.20 & 2.213 & $0.036^{*}$ \\
\hline Co Go & $47-56$ & 52.45 & 3.44 & $44-53$ & 48.80 & 2.09 & 4.053 & $0.001 * *$ \\
\hline Go Gn & $60-72$ & 65.80 & 3.12 & $65-72$ & 68.45 & 2.46 & 2.982 & $0.005 * *$ \\
\hline $\begin{array}{c}\text { AP Max } \\
\text { PNS }\end{array}$ & $42-53$ & 49.10 & 2.92 & $44-52$ & 46.60 & 2.41 & 2.952 & $0.005 * *$ \\
\hline SNA & $76-87$ & 82.45 & 3.44 & $78-83$ & 80.55 & 1.54 & 2.254 & $0.033^{*}$ \\
\hline SNB & $70-81$ & 76.30 & 2.92 & $75-80$ & 77.60 & 1.54 & 1.761 & 0.089 \\
\hline ANB & $3-11$ & 6.10 & 2.51 & $2-4$ & 2.95 & 0.69 & 5.412 & $0.001 * *$ \\
\hline NS Ar & $117-134$ & 122.9 & 4.19 & $129-130$ & 126.55 & 2.42 & 3.374 & $0.002 * *$ \\
\hline S Ar Go & $136-152$ & 144.9 & 5.30 & $129-151$ & 140.50 & 5.02 & 2.727 & $0.010 *$ \\
\hline Ar Go Me & $124-138$ & 130.8 & 4.18 & $116-131$ & 125.95 & 4.43 & 3.525 & $0.001 * *$ \\
\hline Sum & $386-409$ & 398.8 & 7.16 & $373-402$ & 393.35 & 6.06 & 2.598 & $0.013^{*}$ \\
\hline NS Gn & $65-75$ & 70.05 & 3.38 & $66-70$ & 68.40 & 1.20 & 2.060 & 0.051 \\
\hline Pal Mp & $22-42$ & 29.10 & 5.83 & $22-30$ & 25.90 & 2.55 & 2.249 & $0.033 *$ \\
\hline
\end{tabular}

* Sig at $0.05 \%$

** Sig at $0.01 \%$ 
Egyptian

Orthodontic Journal

Table (7): Correlation between P-CV measurements and other studied variables and probability values (both sexes combined).

\begin{tabular}{|c|c|c|c|c|c|c|}
\hline \multirow{2}{*}{ Variables } & \multicolumn{2}{|c}{ PCV2 } & \multicolumn{2}{c|}{ PCV3 } & \multicolumn{2}{c|}{ PCV4 } \\
\cline { 2 - 7 } & $\mathbf{r}$ & $\mathbf{p}$ & $\mathbf{r}$ & $\mathbf{p}$ & $\mathbf{r}$ & $\mathbf{p}$ \\
\hline P-CV3 & 0.909 & $0.001 * *$ & ------ & ------ & ------ & ------ \\
\hline P-CV4 & 0.885 & $0.001 * *$ & 0.896 & $0.001 * *$ & ------ & ------- \\
\hline \hline N ANS & -0.142 & 0.384 & -0.109 & 0.504 & 0.076 & 0.641 \\
\hline ANS Me & -0.305 & 0.055 & -0.368 & $0.019 *$ & -0.205 & 0.205 \\
\hline N Me & -0.067 & 0.681 & -0.103 & 0.527 & 0.131 & 0.421 \\
\hline S Go & -0.312 & 0.050 & -0.111 & 0.495 & -0.033 & 0.839 \\
\hline S Gn & -0.364 & $0.021 *$ & -0.353 & $0.025 *$ & -0.174 & 0.282 \\
\hline Co Gn & 0.091 & 0.577 & 0.114 & 0.484 & 0.330 & $0.038^{*}$ \\
\hline Co Go & -0.261 & 0.104 & -0.230 & 0.154 & -0.008 & 0.959 \\
\hline Go Gn & 0.543 & $0.001 * *$ & 0.541 & $0.001 * *$ & 0.583 & $0.001 * *$ \\
\hline Ap Max -PNS & -0.133 & 0.414 & -0.150 & 0.355 & -0.061 & 0.710 \\
\hline \hline SNA & -0.164 & 0.312 & -0.265 & 0.099 & -0.191 & 0.238 \\
\hline SNB & 0.224 & 0.165 & 0.226 & 0.161 & 0.089 & 0.587 \\
\hline ANB & -0.421 & $0.007 * *$ & -0.541 & $0.001 * *$ & -0.321 & $0.044 *$ \\
\hline NS Ar & 0.539 & $0.001 * *$ & 0.651 & $0.001 * *$ & 0.534 & $0.001 * *$ \\
\hline S Ar Go & -0.403 & $0.010 *$ & -0.371 & $0.019 *$ & -0.305 & 0.055 \\
\hline Ar Go Me & -0.284 & 0.124 & -0.405 & $0.009 * *$ & -0.285 & 0.074 \\
\hline Sum & -0.160 & 0.323 & -0.231 & 0.152 & -0.093 & 0.568 \\
\hline NS Gn & -0.151 & 0.351 & -0.316 & $0.047 *$ & -0.138 & 0.396 \\
\hline Pal Mp & -0.169 & 0.298 & -0.350 & $0.027 *$ & -0.113 & 0.487 \\
\hline
\end{tabular}

* Sig at $0.05 \%$

** Sig at $0.01 \%$ 


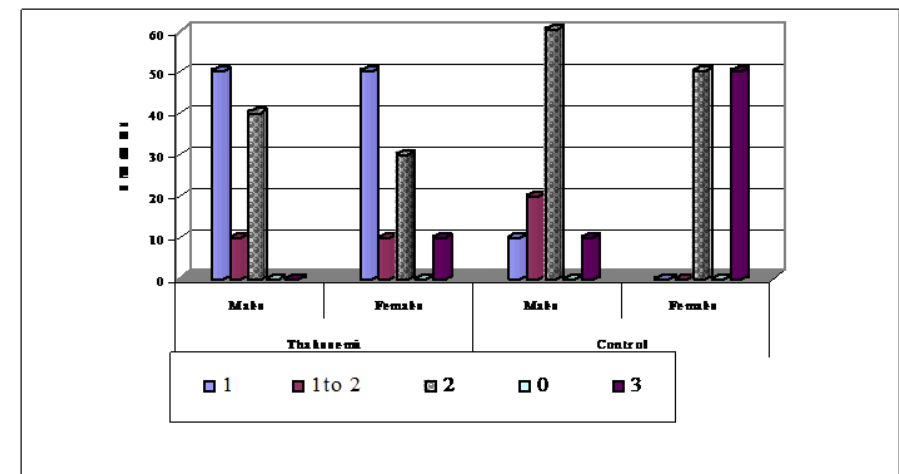

Figure(3): the destribution of the sample by sex, and stages of cervical vertebrae maturation and their percentages.

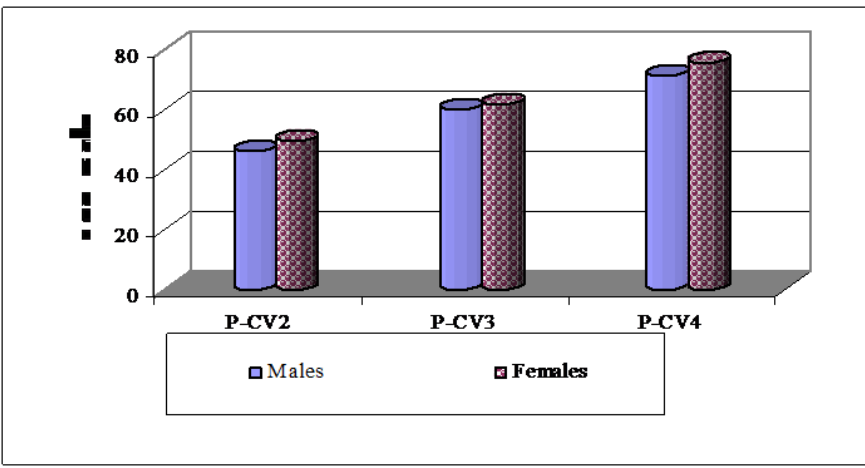

Figure(4): Comparison of P-CV measurements between thalassemia cases in relation to sex

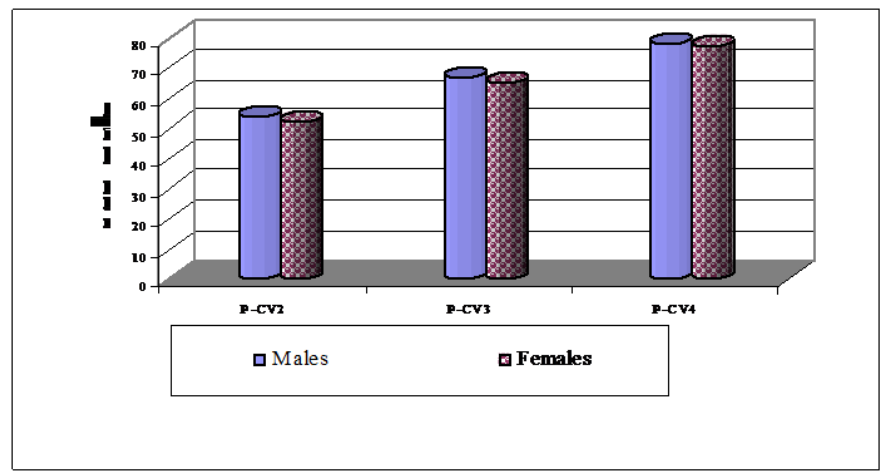

Figure (5): Comparison of P-CV measurements between control in relation to sex 


\section{Egyptian}

Orthodontic Journal

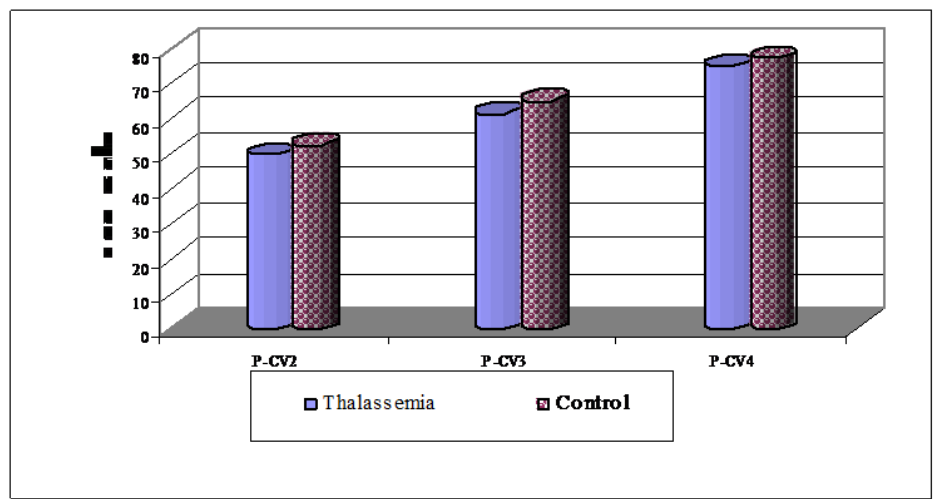

Figure (6): Comparison of P-CV measurements between males of thalssemia and control

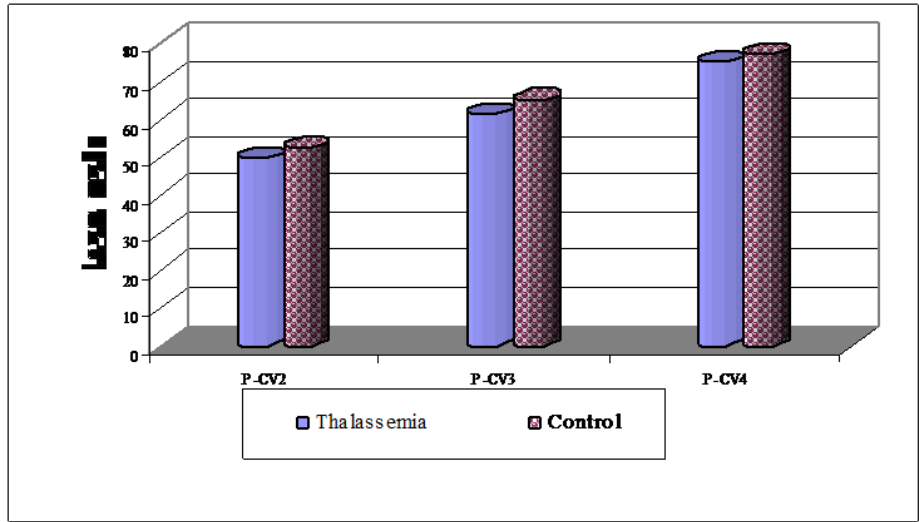

Figure (7): Comparison of $\mathrm{P}-\mathrm{CV}$ measurements between females of thalsssemia and control

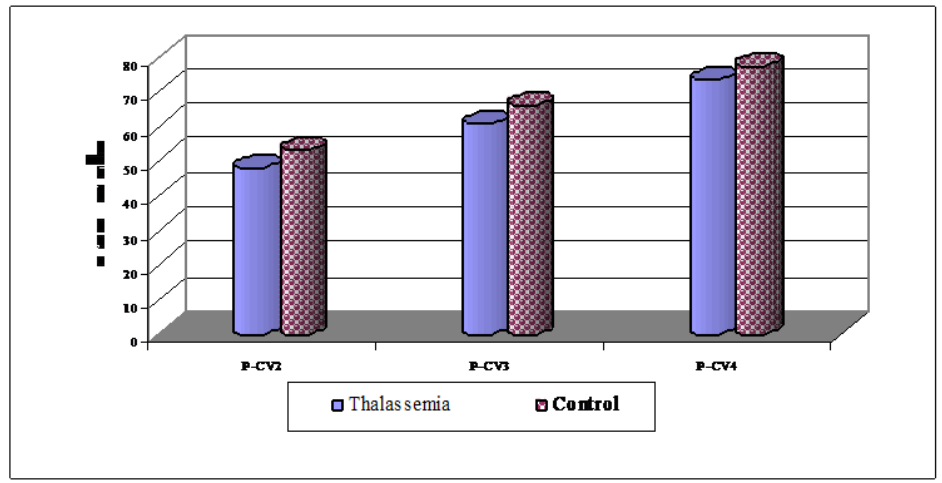

Figure (8): Comparison of P-CV measurements between thalassemic and control groups (both sexes combined 
Egyptian

Orthodontic Journal

\section{DISCUSSION}

Because of individual variation on timing, duration and velocity of growth, skeletal age assessment is essential in formulating viable orthodontic treatment plans. Orthodontists do not necessarily need to know the exact skeletal age of a patient, they need only to know whether the patient will grow and what percentage of growth can be expected.

The cervical spine area, although obvious in most lateral cephalograms, is often neglected by orthodontists and omitted from cephalomertic tracing.

The present study was designed to clarify the effect of $\beta$-thalassemia on the skeletal maturation of the affected children from the cervical vertebrae and the craniofacial alteration without the need for additional radiographs.

Vertebral growth takes place from the cartilaginous layer on the superior and inferior surfaces of each vertebrae ${ }^{25}$. Secondary ossification nuclei on the tips of the bifid spinous processes and transverse processes appear during puberty. Secondary ossification nuclei unite with the spinous proceses when vertebral growth is complete ${ }^{25}$.

Three stages corresponding to 3 different maturational phases in cervical vertebrae (vertebral stages 1 to 3 ) could be identified in thalassemic patient and control subjects in the current study. The vertebral stages 1 through 3 occur in the accelerative growth phase. The prevalence rate of thalassemic patients who presented at stage 1 was $50 \%$ for males and $50 \%$ for females, while at stage 2 , it was $40 \%$ for males and $30 \%$ for females. On the other hand, the prevalence rate of control subjects who presented at stage 2 was $60 \%$ for males and $50 \%$ for females while at stage 3 , it was $10 \%$ for males and $50 \%$ for females. These data clearly demonstrate that the majority of thalassemic patients were at stage 1 through 2. On contrary, stage 2 through 3 , occurred in the majority of control subjects. This means delayed skeletal maturation of thalassemic patients.

In addition, measurements from lateral cephalograms of the cervical vertebrae ( P-CV2, P- CV3 and P-CV4 ) were used to confirm the skeletal maturation stages. The results of the present study revealed that the mean values of P-CV2, P-CV3 and P-CV4 were significantly reduced in the male patients rather than the matching control sample. While female patients showed significant reduction in P-CV3 measurements only.

It should be noted that male thalassemic patients exhibited more delayed skeletal maturation than female patients. The same result was reached by Borgana Pignatt ${ }^{10}$ and Ghobashy ${ }^{26}$. Again and according to the combined 
values, the mean values of all cervical vertebrae in both sexes of thalassemic patients were significantly lower than the mean of the corresponding measurements in control group. This result coincides with Kattamis et al. ${ }^{27}$ and Ghobashy ${ }^{26}$.

Regarding the effect of $\beta$ thalassemia on the craniofacial morphology, it was found that the means of SNA in male patients and both sexes combined were significantly increased than the matching control subjects. This result is in agreement with Goaz and White ${ }^{28}$ and Stewart et al. ${ }^{29}$ who stated that in thalassemic patient, the premaxilla is prominent and that there is protrusion of the middle third of the face. This finding disagrees with Abou El-Ezz ${ }^{30}$ and Ghobashy ${ }^{26}$.

SNB mean was significantly lower in female thalassemic patient than the matching control. This is could be attributed to the significant increase in: lower face height, total face height, gonial angle, sum of posterior angles, Y axis angle and basal plane angle. While in male patients and both sexes combined, there was no significant difference. This result also contradict Abou El-Ezz ${ }^{30}$ and Ghobashy ${ }^{26}$.

The mean of ANB angle was significantly higher in thalassemic patients than the control subjects. This finding supports the results of Abou El-Ezz ${ }^{30}$ and Ghobashy ${ }^{26}$. The increased value of ANB is probably due to either significant low values of SNB or increase of SNA or both.

The mean values of mandibular base length were significantly lower in male thalassemic patients and both sexes combined, and insignificantly decrease in female patients. This finding is in agreement with Ghobashy ${ }^{26}$.

The maxillary base length showed significant increase in female patients and both sexes combined and insignificant increase in male patients hence, caused prominence of the premaxilla. This coincides with Goaz and White ${ }^{28}$ and Van Dis and Langlais ${ }^{31}$.

Cohen and Baty ${ }^{32}$ reported that, there was a remarkable increase in the vertical dimension of the posterior portion of the mandible in thalassemic patients. This is confirmed by the results of the present study where as the mean values of ramus height ( $\mathrm{Co}$ Go ) and posterior face height (S Go ) were significantly increased in males and non significantly increased in female patients. Again in both sexes combined these variables showed significant increase than the matching control sample. 
Egyptian

Orthodontic Journal

The mandible in male thalassemic patients showed tendency to downward and backward rotation as evidenced by significant increase of lower anterior face height and ANB and significant decrease of mandibular length. This finding confirms that reached by Abou El-Ezz ${ }^{30}$ who found significant increase of FH MP, MMP and ANB angles, when compared to normal children.

The mandible in female thalassemic patient showed the same tendency due to significant increase of lower anterior face height, total face height, gonial angle, sum of posterior angles, Yaxis and basal plane angle and significant decrease of SNB angle. This result supports Ghobashy ${ }^{26}$, who stated that the significant increase of the mean values of SN/MP and FH/MP angles may be due to the tendency of the mandible to obtain clockwise rotation in thalassemic patients.

Finally, Pearson's correlation coefficient was performed to detect if a correlation existed among the cervical vertebrae measurements (P-CV2, P-CV3, $\mathrm{P}-\mathrm{CV} 4)$ and between the cervical vertebrae and the variables used in this study (Table7). The results of the present study revealed that there was strong positive correlation among the cervical vertebrae measurements $(\mathrm{P}=0.001)$. This may indicate that they are under strong influence of an intrinsic genetic factor. The correlation also gives valuable informations about which variable of the craniofacial morphology of thalassemic patients related to the cervical vertebrae measurements .

\section{CONCLUSION}

- $\beta$ thalassemia could affect the normal growth of the affected children as evidenced by delayed skeletal maturation and altered craniofacial morphology.

- The appraisal of the stages in cervical vertebral maturation is fairly appropriate for the assessment of individual skeletal maturity on the basis of a single cephalometric observation and thereby determine whether the possibility of potential growth existed. 
Egyptian

Orthodontic Journal

\section{REFERENCE}

1. Modell B. An overview of clinical aspects of thalassemia managnent. Thalassemia today the Mediterraneon Experience. Milano, p.70, 1987.

2. Nienhius AW, Propper RD. The thalassemia disorders of hemoglobin synthesis Hematology of infancy and childhood chapter 24, 1979.

3. Poyton H, Davey KW. Thalassemia: changes visible in radiographs used in dentistry. Oral Surg Oral Med Oral Path 4. 564, 1968.

4. Alagna S, Masala A, Gallisia D. Parathyroid function and bone metabolism in children with beta thalassemia major: effects of sex steroid treatment. Eur J Med 1: 153- 157, 1992.

5. Greep N, Anderson AL, Gallagher JC. Thalassemia minor : a risk factor for osteoporosis. Bone Mine 16:63-72, 1992.

6. Wintrobe MM. Clinical Haematology, $5^{\text {th }}$ ed, Philadelphia Lea and Febiger, p.700-730, 1961.

7. Connell $\mathrm{H}$. the development of oral and facial signs in $\beta$ - thalassemia major. Br Dent J 164:50, 1988.

8. Lynch MA, Brightman VJ, Greenberg MS. Burket's Oral Medicine, Diagnosis and treatment $8^{\text {th }}$ ed. p.756-758, 1984.

9. Caffey J Cooley's anaemia: a review of the reontgenographic findings in the skeleton. Am J Roentg 78: 381-391, 1957.

10. Borgna Pignatti C, De Stefano P, Zanto L, Vullo C, Desanclis V, Melevendi C, Naselli A, Masera G, Terzoli S, Gabutti V, Piga A. Growth and puberty in thalassemia major. An interim report. International Mediterranean conference on endocrine disorders in thalassemia. Cosenza, Italy, May p.7-9, 1992.

11. Kattamis C. Growth in patients with thalassemia. International Mediterranean conference on endocrine disorders in thalassemia. Cosenza, Italy, May p.11, 1992.

12. khaldi F, Beyaoui M, Guemiro F, Messaoud T, Hammou A, Lakhoura R, Ben Naceur B, Farroum S. Growth rate evaluation in Tunisia thalassemic patients. International Mediterranean conference on endocrine disorders in thalassemia. Cosenza, Italy, May p. 31, 1992. 
Egyptian

Orthodontic Journal

13. Ponte G, Divaia P, Lorenzo L, Esposito L. Growth in homozygous $\beta$ thalassemia International Mediterranean conference on endocrine disorders in thalassemia. Cosenza, Italy May p.53, 1992.

14. Hunter WS. The correlation of facial growth with body height and skeletal maturation at adolescence. Angle Orthod 36:44-54, 1966.

15. Taranger $\mathbf{J}$ Hägg U. Timing and duration of adolescent growth. Acta Odontol Scand 38:57-67, 1980.

16. Lewis $\mathrm{AB}$, Garn SM. The relationship between tooth formation and other maturation factors. Angle Orthod 30:70-77, 1960.

17. Tanner JM Growth at adolescence, $2^{\text {nd }}$ ed. Oxford: Blackwell Scientific Publications, 1962.

18. Lamparski DG. Skeletal age assessment utilizing cervical vertebrae (dissertation). Pittsburgh, PA: The University of Pittsburgh; 1972.

19. O'Reilly M, Yanniello GJ. Mandibular growth changes and maturation of cervical vertebrae: a longitudinal cephalometric study. Angle Orthod 58:179-184, 1988.

20. Franchi L, Baccetti T, Mc Namara JA. Mandibular growth as related to cervical vertebral maturation and body height. Am J Orthod Dentofac Orthop 118: 335 -340, 2000.

21. Hassel B, Farman A. Skeletal maturation evaluation using cervical vertebrae. Am J Orthod, Dentofac Orthop 107: 58-66, 1995.

22. Garcia - Fernandez P, Torre H, Flores L, Rea J. The cervical vertebrae as maturational indicators. J Clin Orthod 32: 221-225, 1998.

23. Bench RW. Growth of the cervical vertebrae as related to tongue, face, and denture behavior. Am J Orthod 49:183-214, 1963.

24. Dawson - Saunders B, Trapp R. Basic and Clinical Biostatistics, $2^{\text {nd }}$ ed. Lange Medical Book, Prentice - Hall International Inc 1994.

25. Bradford DS. The pediatric spine. New York: theime, 1985.

26. Ghobashy SA. Study of craniofacial morphology and skeletal maturation in $\beta$-thalassemic patients. Master thesis, Tanta University, 1994.

Volume 27 - June 2005 
27. Kattamis C, Liakopoulou T, Kattamis A. Growth and development in children with thalassemia major. Acta Pediat Scand (Suppl) 366: 111-117, 1990.

28. Goaz PW, White SC. Oral radiology: principles and interpretation. St., Louis. The CV Mosby p.578, 1982.

29. Stewart RE, Barber TK, Troutman KC, Wet SHY. Pediatric dentistry: Scientific foundation and clinical practice. St Louis, Toronto, London: The CV Mosby Co. p. 254, 1982.

30. Abou El-Ezz A M. A study of occlusion and dentoskeletal pattern in a group of Egyptian children suffering from thalassemia. Master thesis, Cairo University, 1993.

31. Van Dis LM, Longlais RR. The thalassemia: Oral manifestation and complications. Oral Surg Oral Med Oral path 62: 229-233, 1986.

32. Cohen MM, Baty JM. Oral manifestations of erythroblastic anemia. J Am Dent Assoc 32: 1396-1403, 1945. 